\title{
Author Correction: Nanoscale bubble domains with polar topologies in bulk ferroelectrics
}

Jie Yin (1), Hongxiang Zong (D), Hong Tao, Xuefei Tao, Haijun Wu (D), Yang Zhang, Li-Dong Zhao (1), Xiangdong Ding, Jun Sun, Jianguo Zhu (D, Jiagang Wu \& Stephen J. Pennycook (D)

Correction to: Nature Communications https://doi.org/10.1038/s41467-021-23863-w; published online 15 June 2021

The original version of this Article misspelled the following number in the Acknowledgements: '52032007', which incorrectly read '52032007, 52032007'. This has now been corrected in both the PDF and HTML versions of the Article.

Published online: 24 June 2021

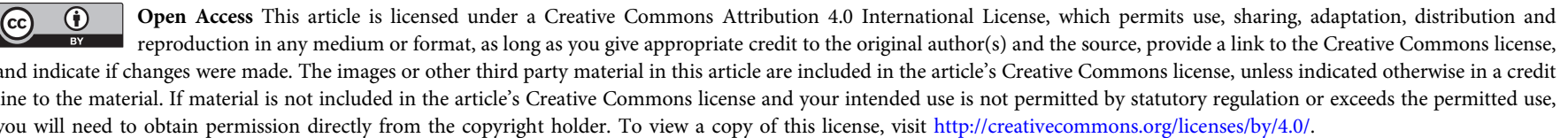
you will need to obtain permission directly from the copyright holder. To view a copy of this license, visit http://creativecommons.org/licenses/by/4.0/.

(c) The Author(s) 2021 